\title{
Le donneur, le receveur et la sage femme. Échanges de cochons à Oksapmin (Papouasie Nouvelle- Guinée)
}

\section{Lorenzo Brutti et Manuel Boissière}

\section{(2) OpenEdition Journals}

Édition électronique

URL : http://journals.openedition.org/jso/1458

DOI : $10.4000 /$ jso. 1458

ISSN : 1760-7256

Éditeur

Société des océanistes

\section{Édition imprimée}

Date de publication : 1 décembre 2002

Pagination : 141-157

ISSN : 0300-953x

\section{Référence électronique}

Lorenzo Brutti et Manuel Boissière, «Le donneur, le receveur et la sage femme. Échanges de cochons à Oksapmin (Papouasie Nouvelle-Guinée) », Journal de la Société des Océanistes [En ligne], 114-115| Année 2002, mis en ligne le 26 mai 2008, consulté le 22 mars 2021. URL : http:// journals.openedition.org/jso/1458; DOI : https://doi.org/10.4000/jso.1458

\section{(c) $(1)(9)$}

Journal de la société des océanistes est mis à disposition selon les termes de la Licence Creative Commons Attribution - Pas d'Utilisation Commerciale - Pas de Modification 4.0 International. 


\title{
Le donneur, le receveur et la sage femme. Échanges de cochons à Oksapmin (Papouasie Nouvelle-Guinée) ${ }^{1}$
}

par

\author{
Lorenzo BRUTTI *, Manuel BOISSIÈRE **
}

\section{RÉSUMÉ}

Le cochon en Papouasie Nouvelle-Guinée joue un rôle important dans la cosmologie des villageois, dans les pratiques agricoles, mais aussi dans les relations d'échange entre groupes sociaux. Dans cet article nous proposons d'étudier, dans une société des Hautes terres, à Oksapmin, d'une part la place du cochon dans la mythologie et dans les cérémonies traditionnelles, puis nous nous penchons sur les pratiques d'élevage (quantité et variétés de cochons élevés, nourriture), pour décrire finalement une forme particulière d'échange faisant intervenir trois personnalités: un donneur, un receveur et un tiers. Cette étude nous permet de proposer une interprétation des relations entre les villageois et leur environnement qui prenne en compte les pratiques agricoles et d'élevage qui y sont menées.

Mots-CLÉs : Papouasie Nouvelle-Guinée, Oksapmin, cochons, porcs, partage, cosmologie, élevage.

\section{Introduction}

J. Barrau s'est passionné pour le rôle et l'usage du cochon en Mélanésie (Barrau, 1973), notam-

\begin{abstract}
Pig plays an important role in the cosmology of Papua New Guinean societies, as well as in the agricultural practices and in the building of relationships between social groups. In this essay we describe the place of pig in the mythology and the traditional ceremonies together with the husbandry practices (quantity and varieties of pigs, feeding practices) of a highland society, the Oksapmin. Furthermore, we illustrate a particular form of exchange where three characters play a role: a donor, a receiver and a third person. The following analysis let us to interpretate the relationships between the humans and their ecosystem according to the agricultural practices and to pig husbandry.
\end{abstract}

KeYwords : Papua New-Guinea, Oksapmin, pigs, pork, sharing out, cosmology, husbandry.

ment à travers l'exemple donné par Rappaport (1970) à propos des Tsembaga. Barrau reprend l'idée selon laquelle, dans le cas des Tsembaga, les cochons peuvent être considérés comme des

1. Cet article a été écrit à quatre mains d'après l'ethnographie recueillie par Lorenzo Brutti entre février 1995 et janvier 2000 et par Manuel Boissière entre septembre et décembre 2001. Lorenzo Brutti a invité à Oksapmin Manuel Boissière, qui souhaitait mener un terrain comparatif en Papouasie Nouvelle-Guinée après ses recherches chez les Yali de Papouasie occidentale.

* Ethnologue à l'ums 1834, CNRS. Le terrain de recherche en Papouasie Nouvelle-Guinée a été possible grâce à une allocation de recherche du ministère de l'Éducation nationale et une autre du programme européen APFT. L'aide logistique du CREDO, du PNGIMR et de la RSPAS a aussi été précieuse. Mais ce travail n'aurait jamais été possible sans la chaleureuse collaboration de la population oksapmin.

${ }_{* *}^{*}$ Ethnobotaniste, Cirad-forêt, TA 10/D Campus de Baillarguet, 34398 Montpellier Cedex 5. Les données réunies par M. Boissière l'ont été grâce à l'obtention d'une bourse de la Fondation Fyssen. 
éboueurs et « [...] nettoient le village et ses abords en mangeant les ordures diverses, y compris les déjections humaines » (Barrau, 1975 : 35). Mais les cochons, comme les hommes, sont surtout des consommateurs primaires (essentiellement de tubercules de taros et de patates douces) et, à ce titre, entrent en compétition pour la recherche d'aliments.

D'autre part, selon Barrau (1990 : 22) la quantité de cochons élevés participe à l'équilibre entre activités humaines et milieu naturel. Une quantité de cochons trop importante crée un déséquilibre. Les hommes sacrifient alors des cochons pour rétablir cet équilibre entre société humaine et écosystème, et ce sont également des «[...] occasions de renforcer les liens d'amitié avec les groupes voisins, de faciliter les mariages, d'évaluer le rapport de forces avec des potentiels alliés ou adversaires $»$.

Le cochon a encore, en Nouvelle-Guinée, une valeur d'échange qui le place au premier rang des richesses d'un village, au même titre que les jardins, au fonctionnement duquel il participe, de manière directe ou indirecte. Malgré son introduction relativement « récente » (5000 ans selon Diamond 1997), la place qu'il occupe dans la plupart des sociétés de Nouvelle-Guinée est centrale (Lemonnier, 1990). Son rôle, outre alimentaire, est également social (paiement de compensations, prix de la fiancée), rituel (usage de la graisse de porc et de diverses parties de son anatomie) et mythique (rôle symbolique du cochon dans les mythes fondateurs). Selon Haudricourt (1972: $38^{2}$ ), «Aux NouvellesHébrides, le cochon élevé et échangé pour obtenir une promotion sociale avait habitué les indigènes à une sorte de transaction monétaire : ils ont accepté facilement le salariat apporté par les Européens, alors qu'en Nouvelle-Calédonie, les échanges limités aux aliments de consommation immédiate expliquent la résistance des populations à accepter l'usage du travail salarié ». Le rôle du cochon dans les échanges est donc révélateur des rapports sociaux. Les changements dont les sociétés de Nouvelle-Guinée font l'objet ont certes atténué l'usage du cochon dans les échanges, le cochon étant de plus en plus remplacé par de l'argent. Il reste néanmoins indispensable à l'élaboration de réseaux entre personnes d'un même groupe linguistique, voire de groupes assez éloignés. Enfin, le cochon est une part inaliénable des pratiques agricoles, la productivité dans les jardins pouvant être fonction notamment du nombre de cochons élevés, et la présence de cochons à proximité des cultures modifiant les rapports entre les villageois.

Le territoire oksapmin est composé de cinq vallées dont la vallée de Trangap, lieu de la recherche, situé à $1600 \mathrm{~m}$ dans la province de Sandaun, non loin de la frontière avec la Papouasie occidentale. L'économie et la société oksapmin ont été profondément transformées par les relations que les Oksapmin entretiennent avec la mine d'Ok Tedi Mining Ltd (OTML, située à Tabubil), cette dernière s'approvisionnant dans les villages avoisinants en produits agricoles (Brutti, 1999 : 189). Les villages se fournissent à Tabubil en produits de consommation et de nouvelles races de cochon ont peu à peu remplacé les anciennes, en gardant souvent les mêmes noms.

Notre propos est de présenter comment le cochon à Oksapmin est sujet à des échanges impliquant sa position dans la cosmologie des Oksapmin, son élevage et ses interactions avec les jardins.

\section{Du partage de porcs et d'autres gibiers dans la mythologie}

Selon la cosmologie oksapmin pré-chrétienne, quand une personne mourait, son âme s'en allait au royaume des morts. Elle passait alors au-dessous du plancher d'écorces de pandanus, à l'endroit où les planches s'interrompent pour laisser la place au foyer, au centre de la maison, car à cet endroit les écorces du pandanus sont plus faciles à soulever. Alors, un chaman montait sur le toit de la maison du mort et commençait à jouer du tambour en appelant les ancêtres pour leur demander de le prévenir quand l'âme serait arrivée à destination. Quand les ancêtres répondaient affirmativement, le chaman prévenait les femmes qui commençaient les lamentations funèbres car l'âme était arrivée saine et sauve à sa demeure éternelle. Mais pourquoi faut-il l'intercession du chaman pour s'assurer du voyage de l'âme dans le monde souterrain ? En effet, ce voyage est long et périlleux. Le chemin mène au royaume souterrain des morts, un endroit comparable aux Enfers de la Grèce antique, ni bon ni mauvais, où les âmes des trépassés mènent une existence semblable à celle de leurs descendants vivants : il y a des villages, des jardins, des forêts, des rivières, des montagnes. Mais l'entrée du monde des morts est gardée par un chien féroce, Deundo, le chien de Dahaprhän. Dahaprhän est une sorte de maître de la terre, un géant moqueur protagoniste de nombreuses aventures dans le panthéon oksap- 
min mais, surtout, il est le créateur du monde des morts.

« Il était une fois une femme, elle était célibataire et elle avait une sœur plus jeune. Un jour cette femme envoie sa petite sœur chercher de l'eau. La sœur se rend à la rivière et remplit sa gourde sans s'apercevoir qu'un têtard était dans l'eau et s'introduit dans le conteneur. C'était Dahaprhän garçon qui s'était transformé en têtard. La petite fille revient et donne la gourde à sa grand sœur qui boit de cette eau et avale l'animal. Soudain elle tombe enceinte. Les gens lui demandent qui est l'homme qui l'a mise enceinte mais elle répond : 'Personne! J'ai bu de cette eau et je suis tombée enceinte'. Elle accouche de cet enfant, Dahaprhän. Le bébé grandit très vite et tout jeune il chasse déjà les oiseaux avec son petit arc. Devenu un jeune homme il va recueillir les fruits de deux arbres. Le premier était l'arbre manghen ${ }^{3}$, Dahaprhän le définit comme plante synonyme du sexe de l'homme. Le second était le fruit de l'arbre umbe ${ }^{4}$ et Dahaprhän le définit comme plante synonyme du sexe de la femme. Dahaprhän disait : 'Moi je peux lire dans les pensées des hommes ; je connais aussi le chemin qui mène au ciel, le monde des âmes mortes en guerre.'

Dahaprhän est devenu un jeune homme grand et fort mais un jour, alors que sa mère est allée chercher des rats, il se transforme en bébé, petit comme un rat et il commence à pleurer. Sa mère laisse tomber la chasse aux rats et vient le consoler. Le bébé pleure beaucoup et alors sa mère lui donne un morceau de sa jupe en fibres végétales, pour jouer. Le bébé la laisse tomber et il se jette à l'eau.

[Suit une série de péripéties ou Dahaprhän entre et sort de l'eau des fleuves tout le long du territoire oksapmin en accomplissant plusieurs prodiges. À la fin il s'installe à Tekin avec deux jeunes hommes trouvés le long de son chemin, qui deviennent ses frères].

Un jour Dahaprhän tombe malade et meurt. Les frères vont lui donner sépulture mais il se réveille et vient s'asseoir dans la maison. Il fait cela plusieurs fois, il meurt et il resurgit. Un jour, pendant qu'il était mort, les deux frères vont à Lembanap et les gens de Lembanap leur disent: 'Votre frère vient ici, il y a bâti sa maison et il a son jardin'. Les frères se demandent : 'mais s'il habite avec nous à Tekin, pourquoi vient-il ici ?'. Alors les deux frères demandent aux gens de Lembanap par quel chemin Dahaprhän vient chez eux.

Et les gens de Lembanap leur montrent le chemin qui était un chemin souterrain. Les deux frères rentrent sous terre et parcourent un large chemin qui s'arrête à un moment, sans issue. Alors ils plantent une flèche pour marquer l'endroit, ils reviennent sur leurs pas et ils sortent du chemin souterrain.

Les deux frères rentrent à la maison et il trouvent Dahaprhän qui dort à côté du foyer où il avait allumé un feu. Alors Dahaprhän soulève les écorces de pandanus à côté du plancher et ils voient qu'il y avait une flèche en dessous, leur marque. Pendant que les deux regardent, Dahaprhän se lève et part aux toilettes. Les deux frères regardent encore et voient leur flèche enfoncée verticalement et ils comprennent ce que faisait leur frère pendant qu'il était mort, il préparait ce chemin souterrain!

Un jour, Dahaprhän tue un cochon, il en donne la moitié aux frères et il dit qu'il va en apporter l'autre moitié à des parents. Alors les frères le suivent en cachette et le voient accrocher la carcasse du cochon à un arbre dans la forêt, la toucher avec un bâton et cette moitié de cochon se transforme en un cochon entier. Après, Dahaprhän en apporte la moitié aux parents et l'autre moitié à la maison en disant à ses frères que les parents ont ainsi compensé son cadeau, en donnant encore une autre moitié de cochon. Alors les frères descendent de la montagne où il s'étaient cachés pour espionner Dahaprhän et il traversent avec le chien la rivière Tekin pour rentrer à la maison. En arrivant à la maison, le chien précède les deux frères et il va saluer son maître. Dahaprhän remarque que le chien est mouillé, alors il lui dit : 'tu n'es pas allé en forêt, tu n'es pas allé chasser, tu m'as espionné'. Ensuite il donne à manger aux deux frères la viande d'opossum. Pendant qu'ils mangent, les deux frères lui disent : 'tu as invité plein de gens et tu leur as donné à manger, on t'a vu'. Alors les trois se disputent.

Un autre jour Dahaprhän dit aux deux frères : ‘je suis malade et j'ai envie de manger de l'opossum, allez chasser de l'opossum !' Et les deux partent à la chasse avec le chien. Mais les deux ne s'en vont pas loin, en haute montagne, ils restent près de la maison pour espionner Dahaprhän. Ils voient Dahaprhän rentrer dans la maison, prendre une liane et en jeter quatre morceaux vers quatre points différents : la montagne Bli, la montagne Tongo, la Montana Areyé, la montagne Alim ${ }^{5}$. Il lie ensemble les bouts de la liane qui étaient près de lui pour les tendre. Soudain des milliers d'opossum arrivent en marchant sur les lianes. Ils viennent à Dahaprhän qui les tue et les entasse. En suite Dahaprhän dit : 'qui ira ramasser les bois à brûler ?' et soudain un grand tas de bois coupé apparaît ; Dahaprhän dit: 'qui ira ramasser les pierres pour le four?' et soudain un grand tas de galets pour le four enterré apparaît ; Dahaprhän dit : 'qui ira chercher les feuilles à cuire ?' et soudain un grand amas de larges feuilles apparaît; Dahaprhän dit: 'qui ira cueillir du taro ?' et soudain un grand monticule de taro apparaît; Dahaprhän dit : 'qui va brûler le poil des opossums ?' et soudain les opossums eux-mêmes se jettent dans le feu et en sortent quand leur poil est brûlé.

Alors Dahaprhän commence à brûler le bois pour chauffer les pierres et quand les pierres sont bien chaudes il dit: 'qui ira ramasser les pierres pour le four de

3. Il s'agit d'une plante Cupaniopsis sp., Sapendaceae, qui servait aussi pour fabriquer les yuan wute, les tisonniers employés lors d'un тити (four enterré) pendant le rituel du sacrifice humain du yuan täl (Brutti, 1997).

4. Trichadenia philippinensis, Flacourtiaceae. La forme du fruit pourrait en effet être rapprochée à la forme du sexe d'une femme.

5. Ces quatre toponymes correspondent aux quatre points cardinaux. 
terre?' et soudain les pierres sortent du feu. Alors Dahaprhän dit : 'qui ira cuire les opossums avec les feuilles et les taros ?' et les opossums eux-mêmes s'enveloppent dans les feuilles et entrent dans le four de terre avec les taros. Une fois que la nourriture est cuite, Dahaprhän dit : 'qui viendra manger avec moi ?' et il déracine une souche d'un arbre guhup ${ }^{6}$ et alors des milliers de gens sortent du trou dans la terre et viennent manger avec Dahaprhän. À cet endroit il y avait un arbre $y a r^{7}$. Les gens empoignèrent des flèches et les enfoncèrent dans la terre. Dahaprhän monta sur l'arbre yar et dit aux gens d'empoigner et tenir bien fermes les flèches enfoncées dans le terrain et il chante : 'kiaham gutohahan baliamgutohahe ${ }^{8}$ '. Il se jette sur les flèches et les flèches rentrent dans son corps et il tombe mort sur le lieu du four. Mais soudain il se relève et il chante: 'Moi je suis un vrai homme, moi je sais lire dans vos pensées', et il va ouvrir le four de terre, il asperge de sel la nourriture et il en donne à manger aux gens mais il en met à côté un peu pour ses deux frères. Ensuite il arrache encore l'arbre guhup de terre et tous les gens s'en vont en rentrant dans le trou qui est à la place de la souche de l'arbre. Une fois les gens partis, Dahaprhän rentre dans la maison. Alors les frères descendent de la montagne où ils s'étaient cachés pour espionner Dahaprhän et ils traversent avec le chien la rivière Tekin pour rentrer à la maison. En arrivant à la maison le chien précède les deux frères et il va saluer son maître. Dahaprhän remarque que le chien est mouillé, alors il lui dit : 'tu n'es pas allé en forêt, tu n'es pas allé chasser, tu m'as espionné'. Ensuite, il donne à manger aux deux frères de la viande d'opossum. Pendant qu'ils mangent les deux frères lui disent : 'tu as invité plein des gens et tu leur as donné à manger, on t'a vu'. Alors les trois se disputent.

Après quelques temps Dahaprhän dit à ses deux frères : 'vous me dîtes que je vole les noix de pandanus, que je vole la nourriture, vous me réprimandez, tout le monde me critique, maintenant on va partir au lieu-dit guhup près de la montagne de Tekin, et vous allez me tuer là !'. Ils arrivent à cet endroit où il y avait deux arbres, l'un de tulip ${ }^{9}$ de $\operatorname{simin}^{10}$. Il était ami de ces deux arbres. Il dit à ses deux frères de se disposer à ses côtés. Il prend deux branches de ces deux arbres, un pour chaque main. Il dit aux deux frères qu'il allait compter jusqu'à trois et qu'à trois ils devaient lui tirer des flèches dessus mais à trois il fit un bond en se soulevant et les deux frères s'entretuèrent. En retombant Dahaprhän alla dans un trou dans la terre en disant: 'Je sais lire dans l'esprit des hommes et des femmes, dorénavant je vais prendre de cette façon les âmes des hommes et des femmes qui meurent !'. Il descendit dans la galerie sous terre avec les âmes de ses deux frères et il laissa la garde de l'entrée à son chien Deundo. L'accès au monde souterrain se trouve à Lembanap, au lieu-dit Lemuap, un endroit tabou, où il y a l'entrée d'une caverne entourée de sagou, et une rivière de couleur pourpre que l'on appelle aussi Lemwap ».

Disit Hilin, Tambanaha 23/12/1995

Dans l'agiographie de Dahaprhän on trouve, pour la première fois dans le panthéon Oksapmin ${ }^{11}$, le thème du partage et de l'échange, notamment de l'échange de nourriture animale. En fait, si l'échange de nourriture végétale (tubercules) n'est pas un véritable échange mais plutôt un don ou une entraide commune et presque quotidienne, le partage de nourriture animale présente toujours des caractéristiques exceptionnelles, souvent cérémonielles. Dahaprhän est le maître de la terre, l'initiateur du chemin des morts, qui multiplie la nourriture et la donne à ses frères. Il est important ici de remarquer que l'apparente neutralité de l'échange où personne ne gagne, car un don est compensé par un contredon équivalent, souligne aussi l'importance du partage du porc et de la nourriture animale en général comme stratégie pour nouer des alliances et augmente la réputation d'un grand homme. Dahaprhän est magnanime et donne de la nourriture à ses frères et aux gens qu'il convie aux festins. Nous retrouvons dans le complexe mythique de Dahaprhän plusieurs éléments caractéristiques de la tradition christique. Parmi ces multiples correspondances, celle qui nous intéresse le plus dans ce contexte est la multiplication et le partage de la nourriture, témoignage qui est retrouvé aussi dans les Évangiles et qui inscrit Dahaprhän et le Christ dans la même lignée de sauveurs qui donnent la nourriture aux frères et aux fidèles, mais aussi de psychopompe car l'un et l'autre ouvrent la voie aux chemins de l'au-delà. Dans la tradition oksapmin, la référence chtonienne est bien sûr plus présente que dans la religion chrétienne, fondée par contre sur le sauveur. L'accès à l'au-delà n'est pas gagné par les œuvres accomplies de son vivant en suivant la morale religieuse mais plutôt avec le sacrifice d'un être transitionnel, le cochon, qui devient le substitut de vie, le complément humain qui peut se substituer à l'âme de l'homme et la libérer. Le cochon continue alors à jouer le rôle de l'alter-ego de l'homme, dans le monde des morts comme il le fait aussi dans le monde des vivants.

6. guhup $=$ Dicksonia sp., Dicksoniaceae.

7. yar en pidgin, darap en oksapmin (Casuarina oligodon, Casuarinaceae).

8. Comme il arrive souvent dans le cas des formules magiques, l'auteur n'a pas fourni de traduction pour cette phrase.

9. Tulip en pidgin, yépé en oksapmin (Gnetum gnemon, Gnetaceae).

10. Garcinia sp., Clusiaceae.

11. Comme tous les informateurs s'accordent à le dire, l'épopée de Dahaprhän est chronologiquement plus ancienne de celle de Yuan-ku, l'esprit féminin ancestral régénérateur de l'univers et apporteur de culture. 
Dans le monde des vivants, en fait, le cochon entre en jeu dans la plupart des cérémonies traditionnelles comme porc, donc mort, en termes de portion de viande mais aussi en tant que cochon, donc vivant, ce qui aurait permis de plus longs déplacements et des échanges cérémoniels sur d'importantes distances (Strathern, 1971; Lemonnier, 1990). Lors du rituel en l'honneur de Yuan $-k u^{12}$, des cochons étaient tués et mangés parallèlement au sacrifice humain, pour souligner l'importance de cet animal, non pas comme substitut mais comme complément, équivalent de l'homme (Brutti, 1997). Cette complémentarité est aussi retrouvée lors de la descente aux enfers de l'âme d'un défunt, quand les proches du mort sacrifient un cochon pour que le Cerbère, qui garde l'accès à l'entrée du monde souterrain, dévore l'âme de l'animal et laisse passer celle de l'homme.

\section{Les cochons des Oksapmin}

Partout dans les hautes terres de NouvelleGuinée, le cochon, domestique ou sauvage, est le bien traditionnel le plus précieux et, avec le chien, le seul animal traditionnellement élevé. Les activités d'élevage alimentaire à Oksapmin se résument à l'élevage du cochon domestique. Quelques rares poulets ont été introduits pendant les dernières décennies suite aux contacts avec les Européens, mais ce type d'élevage a obtenu un succès modéré. Les seuls partisans convaincus ont été les Adventistes du Septième Jour qui, pour des raisons liées à leur religion, vivent dans des camps délimités par des barrières, n'élèvent pas de cochons et ne consomment pas de viande de porc. De surcroît, la tentative faite par les fonctionnaires australiens d'introduire du bétail (buffles et brebis) comme facteur d'intensification s'est heurtée à des obstacles importants tels que :

- l'étrangeté de ces animaux pour les populations locales ;

- le coût de leur transport par avion ${ }^{13}$

- la difficulté d'utiliser la traction animale sur des parcelles non dessouchées et souvent en forte pente.

Comprendre le rôle et la place du cochon (täp) dans la société oksapmin et son importance pour l'agriculture (part de la production des jardins qui revient aux cochons) implique l'étude d'un certain nombre de pratiques et de connaissances liées à son élevage : construction de barrières ou de porcheries, nourriture pour les cochons.

\section{Quantité de cochons à Mekiawa}

Avant le contact avec la société des Blancs, un homme riche était un homme qui pouvait avoir un cheptel d'au moins une dizaine de cochons. Un homme riche en cochons est appelé kamehän. Quand ses truies mettent bas, le kame- hän doit distribuer ${ }^{14}$ une partie de la portée à ses parents consanguins et affins pour garder de bonnes relations, surtout avec sa belle-famille, et ne pas avoir la réputation de elua- hän, (avare).

Les cochons, à travers les réseaux d'échanges, passent facilement d'un propriétaire à l'autre, ce qui rend difficile leur dénombrement. Nous avons compté le nombre de cochons élevés par les habitants de deux hameaux du village de Mekiawa: Tambanaha et Dakaleng. Notre étude diachronique s'étale sur six ans, de 1995 à 2001 (voir tableau 1).

TABLEAU 1. - Recensement de cochons à Mekiawa (1995 à 2001); sources : L. Brutti et M. Boissière.

\begin{tabular}{|c|c|c|c|c|}
\hline $\begin{array}{c}\text { Nombre } \\
\text { de foyers }\end{array}$ & $\mathbf{1 9 9 5}$ & $\mathbf{1 9 9 7}$ & $\mathbf{1 9 9 8}$ & $\mathbf{2 0 0 1}$ \\
\hline 10 & 25 & 19 & 21 & 38 \\
& $(11 \mathrm{M} ;$ & $(7 \mathrm{M} ;$ & $(8 \mathrm{M} ;$ & $(23 \mathrm{M} ;$ \\
$14 \mathrm{~F})$ & $12 \mathrm{~F})$ & $13 \mathrm{~F})$ & $15 \mathrm{~F})$ \\
\hline
\end{tabular}

Sur un total de dix foyers, on constate une forte baisse du nombre de cochons, à mettre en relation avec la sécheresse de 1997, due à l'effet El Niño, et avec la famine qui s'en est suivie. Les Oksapmin ont alors consommé une partie de leur cheptel porcin, aujourd'hui largement reconstitué (voire même augmenté par rapport aux données de 1995).

Ces résultats sont néanmoins soumis à évolution, le nombre de cochons échangés, vendus ou tués étant important, lié à un événement ou à la seule volonté des propriétaires. En quelques jours, une partie du cheptel peut se déplacer vers d'autres hameaux de la vallée, ou s'enrichir de nouvelles bêtes laissées en dépôt ou offertes en compensation.

12. Toutes les trois générations, un rituel de fertilité culminant dans un sacrifice humain était célébré à Oksapmin pour que de la déesse ancestrale assure la fertilité des jardins, l'abondance du gibier et la prospérité des humains.

13. L'avion et l'hélicoptère sont les seuls moyens d'accès à Oksapmin, à part les pistes pédestres.

14. Nous verrons plus loin la façon dont ce partage est encore fait de nos jours. 


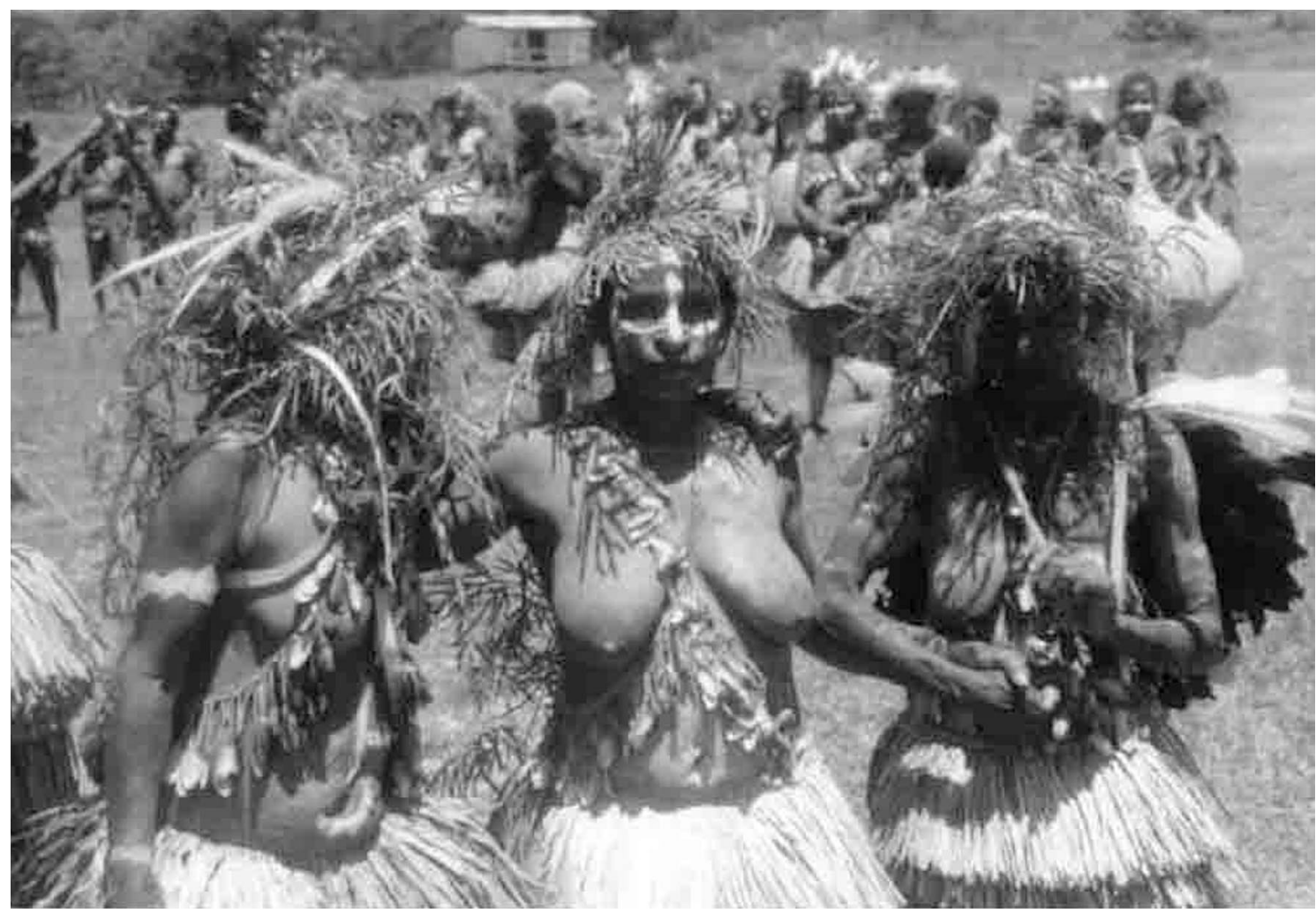

Рното 1. - Trois femmes en parure cérémonielle lors des célébrations pour la fête de l'indépendance. Les filets font partie des prix de la fiancée. Trangap-Oksapmin, 1995 ( Lorenzo Brutti).

Tous les gens interrogés s'accordent pour dire que les races de cochons présentes à ce jour sont nouvelles, même si certaines reprennent les noms de races anciennes élevées par les ancêtres, et perdues depuis.

Nous avons dénombré, à Oksapmin, neuf types de cochons (deux n'existent plus), le plus souvent importés d'Australie, dont les noms reprennent ceux des types locaux.

- bok kat ket / tulboh : cochons noirs et blancs, en deux ou trois bandes transversales.

— täp näp / ku: cochon entièrement noir.

- nau / awam : cochons à bandes brunes.

- tohtoha : cochons à taches brunes et noires.

- ases : cochons à rayures longitudinales noires et brunes.

- toha : cochon entièrement blanc.

- sungwe : cochon entièrement brun.

- koliamo : cochon de n'importe quelle couleur, mais sans queue. Cette race n'existe plus.

- abalia : cette race n'existe plus et les informateurs ne se souviennent plus de sa couleur.

\section{Le triste destin des cochons}

Un cochon adulte peut valoir entre cent cinquante et trois cents kina (en 1996) et même plus s'il s'agit d'une truie, car elle représente un investissement à des fins de reproduction.

Les cochons domestiques sont destinés principalement à la consommation. Ancienne devise coutumière à l'époque pré-contact, les cochons entrent encore en jeu lors du commerce intertribal, des compensations relatives à des accusations de sorcellerie, des compensations de guerre ou en cas de meurtre, mais surtout du paiement des compensations de mariage ${ }^{15}$ (photo 1 ). Les femmes s'occupent de l'élevage des cochons avec un degré de soin comparable à celui qu'elles offrent à leurs enfants. Il est rare mais encore possible de voir des femmes donner le sein aux porcelets (photo 2), alors que les animaux adultes sont nourris de tubercules, notamment de patates douces, une à deux fois par jour, en début et en fin de journée. Les femmes s'approchent des enclos à cochons chargées de leurs filets remplis de tubercules et s'accroupissent pour commen- 


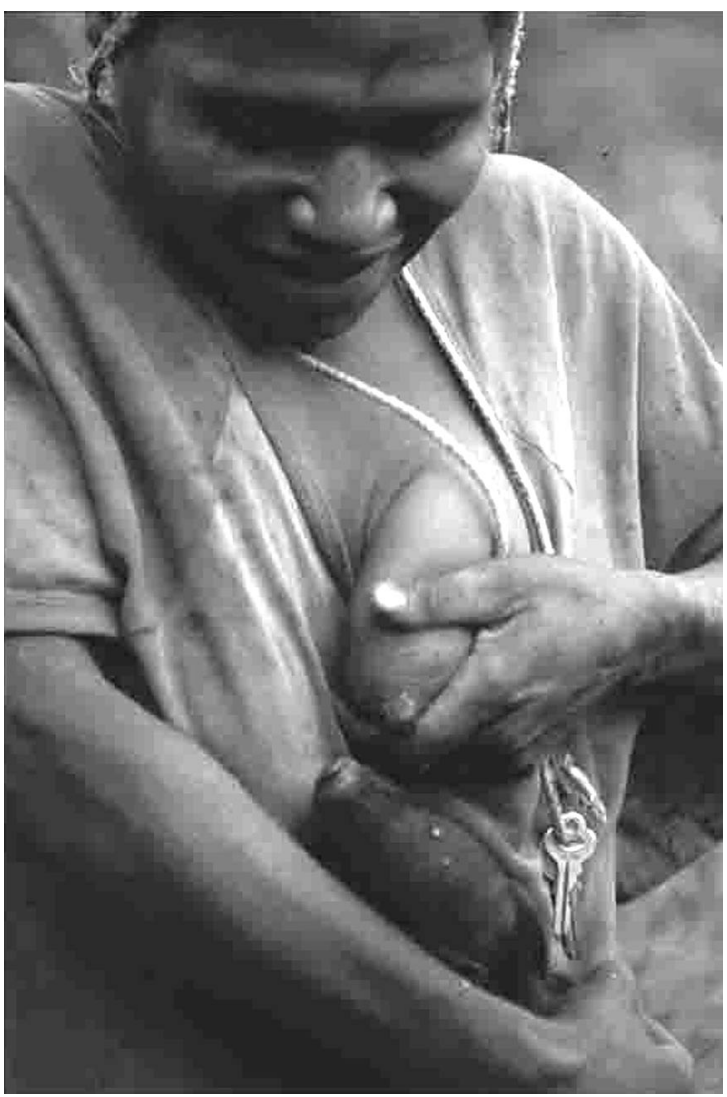

Pното 2. - Femme oksapmin donnant le sein à un porcelet Trangap-Oksapmin, 1998 ( Lorenzo Brutti).

cer à couper des patates douces en morceaux à l'aide d'un outil fabriqué avec un tibia de casoar, mais aujourd'hui de plus en plus avec des couteaux.

Les cochons ont trois destins possibles. Les truies sont en général destinées à la reproduction, sauf en cas de besoin ou de productivité trop faible, et elles sont alors tuées. Les truies sont fécondées par les mâles domestiques mais aussi par les mâles sauvages. Selon Dwyer (1996 : 488), la fécondation de truies par des mâles domestiques n'intervient que dans les régions de haute altitude, où les cochons sauvages sont rares voire absents. Mais dans le cas d'Oksapmin, on trouve suffisamment de cochons sauvages pour que des truies soient souvent laissées en forêt en période fertile. Cela a le double avantage de les rendre gravide et d'amener l'éventuel mâle sauvage à proximité des habitations et d'en faire ainsi un gibier facile. Les mâles peuvent être utilisés en tant que reproducteurs ou castrés ${ }^{16}$ et élevés dans le but de les engraisser pour les vendre ou les consommer directement.

Nous avons fréquemment observé la formation de marchés informels à l'occasion de la vente de parties de porcs qui venaient d'être abattus. La connaissance de la tenue de telles ventes se fait par le bouche à oreille et, très rapidement en quelques heures, il ne reste plus de morceau à acheter. Nous avons pu comparer le coût d'un cochon vendu sur pied et celui d'un porc vendu en morceaux. Il semble économiquement beaucoup plus avantageux de vendre un cochon sur pied qu'en morceaux. La question que l'on peut alors se poser est pourquoi vendre des morceaux alors qu'à moindre effort on gagne plus ?

Nous pensons qu'il n'existe pas une seule réponse à cette question. Pour chaque cas, selon les stratégies que développent les villageois, les explications données à la tenue de ces ventes sont différentes.

D'abord, si un cochon a été blessé, qu'il est malade ou qu'on veuille s'en débarrasser parce qu'on ne peut plus le nourrir ou parce qu'il a saccagé un jardin, il arrive qu'on ne trouve pas d'acheteur pour le cochon sur pied, son prix étant assez important. On est alors obligé de le vendre en morceaux. Le prix de chaque morceau étant relativement faible, de nombreuses personnes viendront acheter et la totalité du porc sera bientôt vendue. Une autre raison peut venir de la volonté de garder une partie du porc pour sa consommation personnelle et de vendre alors ce qui reste pour faire quand même un petit bénéfice. Mais on ne peut négliger une autre raison, plus symbolique : le désir de participer, même à perte, au commerce «moderne », selon l'idée que s'en font les Oksapmin.

\section{Élevage des cochons à Oksapmin}

Les relations de la société oksapmin avec l'élevage et l'échange de cochons a changé depuis quelques années, notamment sous l'influence de techniciens du gouvernement (Département de la Santé). Avant 1995, époque des premiers grands enclos, les cochons vivaient à proximité des villages. Dans la journée ils étaient sortis,

16. Selon Dwyer (1996 : 493), pour maintenir des populations de cochons domestiques à haute altitude, les populations doivent garder quelques verrats, mais en petit nombre à cause du coût pour les élever. La majorité des mâles est donc castrée. A Oksapmin, cette opération a également pour but de ne pas mettre en concurrence trop de verrats dans le même enclos, ce qui pourrait provoquer des luttes. Ce sont toujours les hommes qui s'occupent de l'opération; ils coupent le sexe, les testicules et les défenses du cochon. Les testicules des cochons sont données à manger aux femmes pour qu'elles tombent plus facilement enceintes. Il est par contre interdit aux hommes de manger les testicules des cochons. Même s'il n'y a pas de risque particulier de contracter une maladie au cas où l'on brise ce tabou, on risquerait lors de la prochaine sortie de chasse qu'un cochon sauvage mange les testicules de l'homme fautif. 
leur patte liée à une corde et on les attachait ainsi à un arbre ou un piquet. Le gouvernement a expliqué quels étaient les problèmes sanitaires liés à ce type d'élevage et a incité les Oksapmin à changer leurs habitudes.

À Mekiawa, les premiers villageois à avoir pensé à délimiter un espace réservé exclusivement aux cochons ont été aidés par le Département de l'Agriculture qui leur a fourni une partie des grillages pour faire les enclos. D'autres villageois ont aidé à construire les clôtures, et l'usage du futur enclos a ainsi été défini : sont reconnus propriétaire de l'enclos les villageois qui ont le plus investi, en temps et en argent, dans leur construction. Les villageois qui ont aidé au financement dans une moindre mesure, ou à la construction des barrières, sont dispensés de payer un droit aux propriétaires pour placer leurs cochons dans les enclos. En revanche, les personnes qui n'ont pas aidé ou qui se sont installées dans le village récemment doivent payer un droit pour utiliser l'enclos pour leurs cochons.

Plusieurs grands enclos ont vu ainsi le jour en quelques années, bannissant toute pratique agricole de vastes espaces dont la terre était peu fertile. Un second avantage de ce type d'élevage est qu'il permettait de ne plus construire de barrières autour des nouveaux jardins. Les règles concernant les barrières de jardins se sont reportées sur les clôtures à cochons, le propriétaire du jardin le plus proche d'une clôture brisée et le propriétaire du cochon ayant traversé cette clôture, doivent la réparer ensemble.

Dans d'autres sociétés de Nouvelle-Guinée, on a pu observer de nombreux types de barrières différents, liées ou non à l'élevage des cochons, chez les Karuka (Steensberg, $1980: 123$ ), jusqu'à six chez les Yali (Boissière, 1999 : 273). Chez les Oksapmin, outre les nouvelles barrières en grillage, on ne trouve que trois types de barrières, dont deux seulement liées à l'élevage du cochon. La première est le récent enclos à poulets, fait de fines et hautes tiges liées ensembles: nel dohe (clôture à oiseaux). Les deux autres, pré-contact, se distinguent par la position du bois qui les composent : dans le cas de la barrière uha (photo 3 ), des planches sont plantées verticalement dans le sol et reliées par une tige horizontale. C'est le type de barrière le plus fréquent actuellement. Dans le cas de la barrière dohe (photo 4), des troncs sont allongés horizontalement et retenus par des poteaux, tam. On trouvait souvent ce type de barrière autour des jardins, avant la construction des premiers enclos à cochons.

Lorsque nous avons interrogé nos informateurs sur les raisons de l'usage d'un type de bar- rière plutôt qu'un autre, ils nous ont répondu que dans le cas de dohe, le bois était plus difficile à trouver que dans celui de uha. Il faut en effet comprendre qu'à Oksapmin, la végétation s'est raréfiée dans le fond de vallée. Il ne persiste de bois d'Araucaria ou de Casuarina que dans des secteurs très localisés de la vallée. Construire une barrière à partir de troncs entiers suppose aller chercher la matière première de plus en plus loin pour réaliser quelques mètres de barrières seulement. En revanche, on trouve toujours suffisamment de bois à proximité de la future barrière, même s'il n'a pas la qualité requise pour une barrière de type dohe. On pourra en faire des planches utilisées pour les barrières uha. Ainsi, sans vouloir développer une réflexion déterministe sur l'usage des barrières, on peut dans le cas présent expliquer la perte de certaines connaissances par la diminution de la richesse du milieu naturel. Les jeunes Oksapmin auxquels nous nous sommes adressés ne se souvenaient même plus du nom de la barrière dohe.

Si les enclos à cochons prennent une importance de plus en plus déterminante dans le paysage oksapmin, de nombreux hameaux n'en ont toujours pas, soit par manque de place pour construire un enclos suffisamment grand, soit par l'absence de personnalités intéressées par ce nouveau type d'élevage. Les propriétaires lient alors leurs cochons à des piquets pendant la journée, mais les laissent souvent en liberté lorsqu'ils sont encore petits, estimant qu'ils ne peuvent pas occasionner beaucoup de dégâts. Les deux formules coexistent donc, souvent sujettes, l'une et l'autre, à des conflits sur les dégâts provoqués par les cochons ou sur les droits à payer pour l'usage d'un enclos à cochons.

Les clôtures remontent en général jusqu'à proximité des habitations. En bordure de l'enclos, non loin des maisons, chaque propriétaire de cochons construit une porcherie, lieu où les cochons sont nourris, le matin et le soir, et où ils dorment (photos 5, 6, 7). Les truies mettent bas, en revanche, dans des zones retirées de l'enclos, souvent accessibles au propriétaire seulement. La truie se construit alors avec des herbes (Imperata cylindrica) une niche dans laquelle elle se cache avec sa portée pendant quelques semaines. Le terme täp ap ou maison de cochons, désigne à la fois la porcherie et la niche où les truies s'abritent.

\section{De la nourriture pour les cochons}

Comme l'expliquait J. Barrau (1973 : 42), le lien entre les hommes et les cochons passe par la 


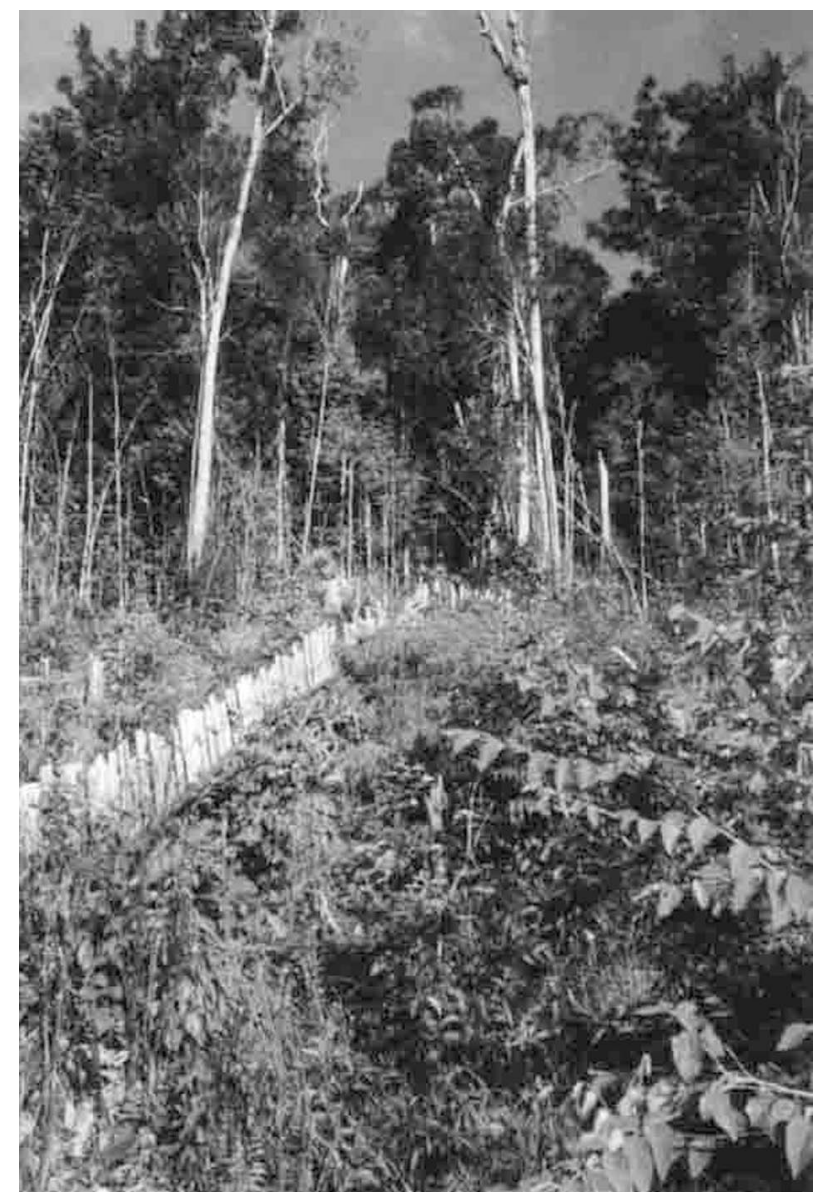

Рното 3. - Les barrières $u h a$, faites de planches plantées verticalement dans le sol. Trangap-Oksapmin, 1996. (Lorenzo Brutti).

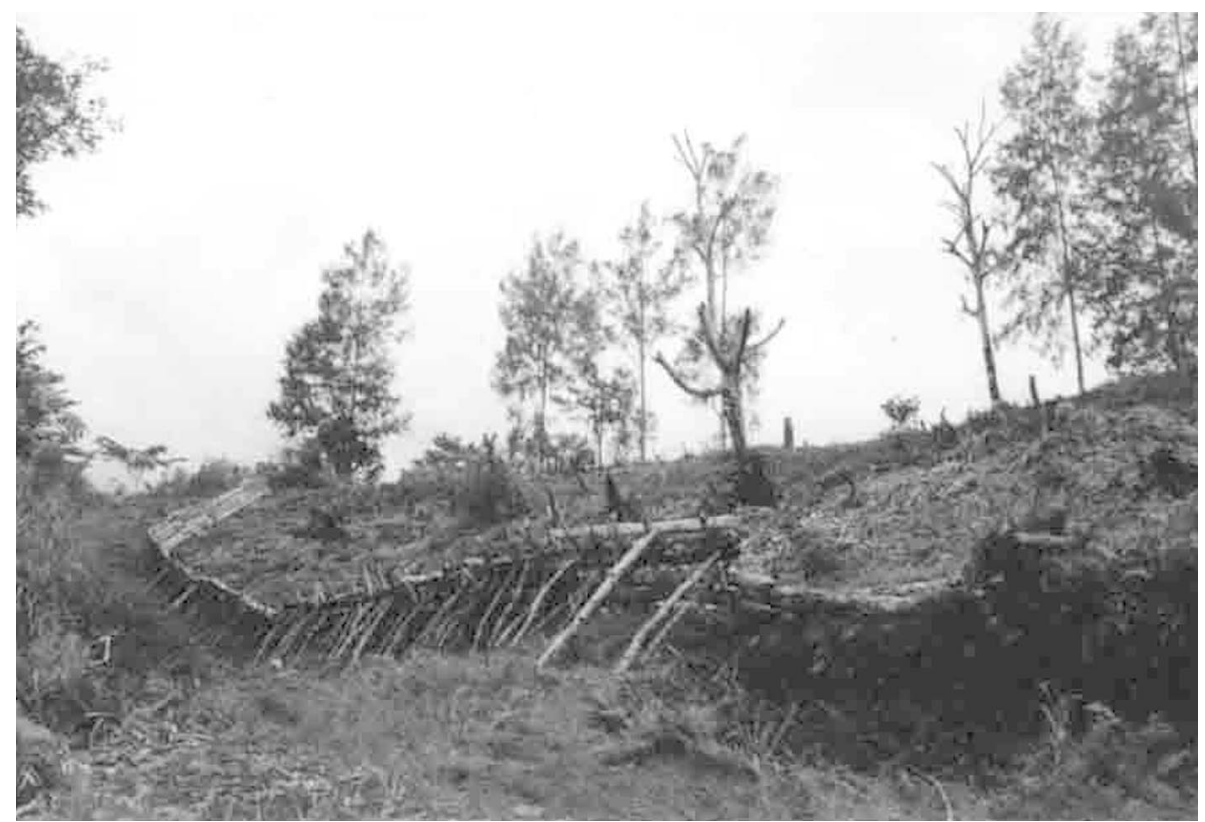

Рното 4. — Les barrières dohe, faites de troncs allongés. Trangap-Oksapmin, 1996. (Lorenzo Brutti). 

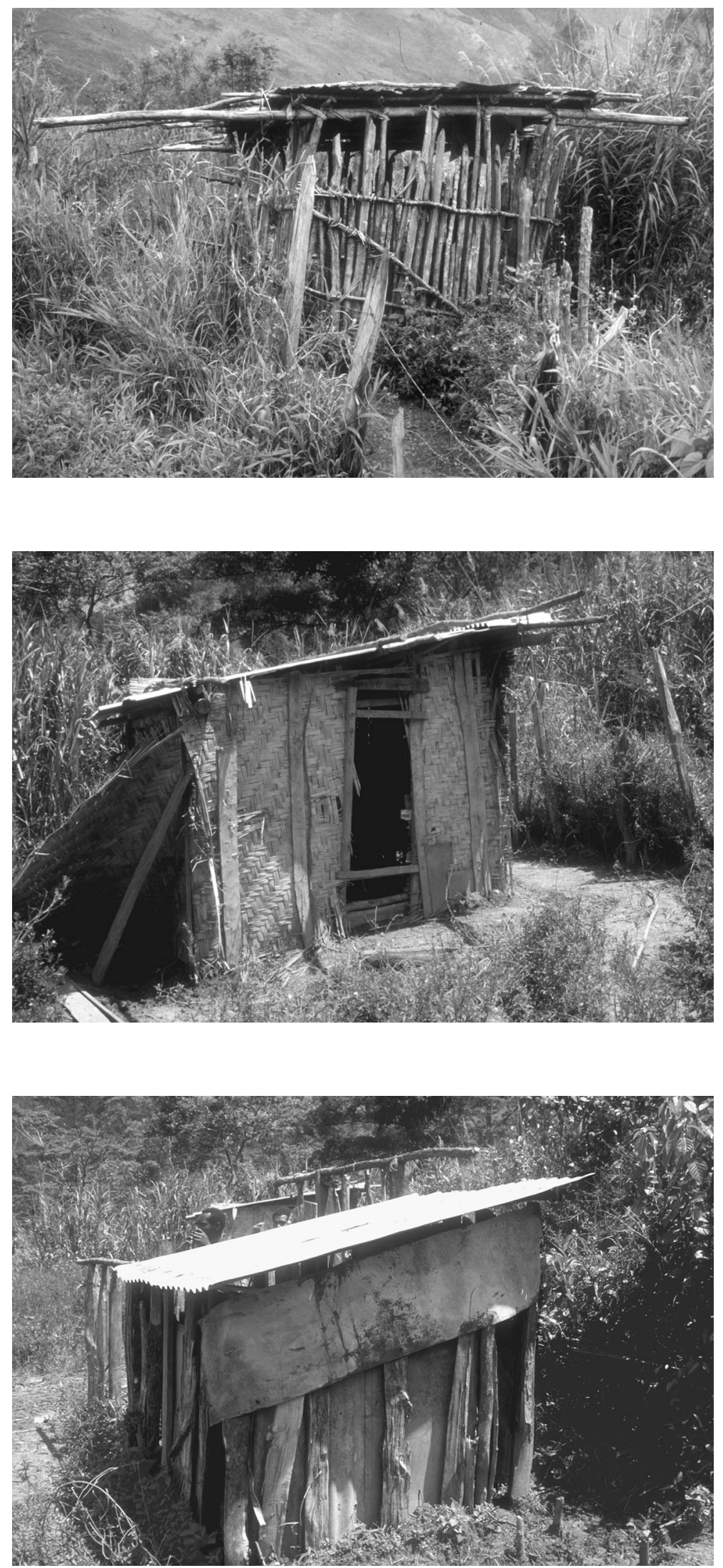

Рното 5, 6 et 7. - Différents types de porcheries. Trangap-Oksapmin, 2001. (Manuel Boissière). 
compétition pour la nourriture. Cette compétition a provoqué l'apparition des clôtures autour des jardins, puis autour des cochons eux-mêmes. Rappaport (1970 : 3) écrit : « La relation entre les gens, les cochons et les jardins est équilibrée. Cet équilibre opère de façon directe pour protéger les gens du possible parasitisme et de la compétition avec leur cochons et de façon indirecte pour protéger l'environnement en aidant à la conservation de larges aires de forêt vierge et en assurant une gestion adéquate de la culture des jachères en forêt secondaire ${ }^{17}$.

L'élevage du cochon est donc un élément important de l'agriculture, autant par les incursions plus ou moins provoquées qu'ils font dans les jardins (Dwyer,1990) que par la part de production des jardins qui leur est réservée. Il était donc important d'étudier la manière dont les Oksapmin nourrissent leurs cochons et la quantité de nourriture qui leur est distribuée chaque jour. La création des enclos à cochons et surtout des porcheries à proximité des maisons a permis de généraliser l'habitude de nourrir les cochons deux fois par jour: le matin et le soir. Les cochons reviennent le soir dans les porcheries et reconnaissent uniquement le cri de leur propriétaire. Comme on peut le voir sur le tableau 2, il arrive que le propriétaire ne nourrisse pas son cochon de la journée ou bien uniquement une fois par jour. Tout dépend de la manière dont il l'élève. Un cochon qui reste en liberté dans la zone clôturée va trouver une partie de sa nourriture lui-même, en fouillant le sol à la recherche de tubercules. S'il est laissé toute la journée la patte liée par une corde, il trouvera moins facilement de la nourriture et devra être nourri plus régulièrement et copieusement. La quantité moyenne de nourriture donnée par cochon et par jour est très variable et dépend de la richesse du propriétaire en jardins (et donc son accès à la nourriture pour les cochons) et de la situation du cochon lui-même. Cette quantité peut varier de 0.88 à 5.5 kgs par cochon et par jour. Certaines personnes nourrissent régulièrement leurs cochons une fois par jour, d'autres seulement lorsqu'ils ont de la nourriture à leur donner. Dans ce dernier cas, il est important que les cochons soient dans un milieu relativement ouvert où ils puissent toujours trouver de quoi se sustenter eux-mêmes.

On donne en général des patates douces crues aux cochons. Lorsque le cochon est encore jeune, on peut cuire ces patates avant. Les jours de marché, les propriétaires qui n'ont pas ou plus de jardin vont essayer de trouver des restes de légumes non consommables pour les hommes. Par exemple des feuilles de choux ou de brocoli, qui sont alors vendues au sac aux propriétaires. C'est ce qui apparaît précédé d'une étoile $\left(^{*}\right)$ dans le tableau 2.

TABLEAU 2. Quantité quotidienne (kgs) de nourriture donnée aux cochons (source : M. Boissière).

\begin{tabular}{|c|c|c|c|c|c|c|c|c|c|c|}
\hline $\begin{array}{c}\text { Propriétaires } \\
\text { des } \\
\text { cochons }\end{array}$ & $\begin{array}{c}07 / 12 / 01 \\
\mathrm{am}\end{array}$ & $\begin{array}{c}07 / 12 / 01 \\
\mathrm{pm}\end{array}$ & $\begin{array}{c}08 / 12 / 01 \\
\mathrm{am}\end{array}$ & $\begin{array}{c}08 / 12 / 01 \\
\mathrm{pm}\end{array}$ & $\begin{array}{c}09 / 12 / 01 \\
\mathrm{am}\end{array}$ & $\begin{array}{c}09 / 12 / 01 \\
\mathrm{pm}\end{array}$ & $\begin{array}{c}10 / 12 / 01 \\
\mathrm{am}\end{array}$ & $\begin{array}{c}10 / 12 / 01 \\
\mathrm{pm}\end{array}$ & $\begin{array}{c}11 / 12 / 01 \\
\mathrm{am}\end{array}$ & $\begin{array}{c}11 / 12 / 01 \\
\mathrm{pm}\end{array}$ \\
\hline 1 & $\mathrm{X}$ & $\mathbf{6}$ & $\mathrm{X}$ & $\mathrm{X}$ & $\mathbf{5}$ & Rien & $\mathrm{X}$ & $\mathrm{X}$ & $\mathbf{8}$ & $\mathbf{6}$ \\
\hline 2 & $\mathrm{X}$ & $\mathbf{4}$ & $\mathrm{X}$ & $\mathrm{X}$ & $\mathbf{5}$ & 4 & Rien & $\mathbf{2}$ & Rien & Rien \\
\hline 3 & $\mathrm{X}$ & $\mathrm{Rien}$ & $\mathrm{X}$ & $\mathrm{X}$ & $\mathbf{5}$ & Rien & $\mathbf{9}$ & 3 & $\mathrm{X}$ & $\mathrm{X}$ \\
\hline 4 & $\mathrm{X}$ & $\mathrm{Rien}$ & $\mathbf{9}$ & 9 & 4 & Rien & $\mathrm{X}$ & $\mathrm{X}$ & $\mathbf{9}$ & Rien \\
\hline 5 & $\mathbf{5}^{* *}$ & $\mathbf{3}$ & $\mathbf{7}$ & 9 & Rien & $\mathbf{3}$ & Rien & $\mathbf{4}$ & Rien & $\mathbf{4}$ \\
\hline
\end{tabular}

\begin{tabular}{|l|l|l|l|l|l|l|l|l|l|}
\hline $\begin{array}{c}\text { Propriétaires } \\
\text { des cochons }\end{array}$ & $\begin{array}{l}12 / 12 / 01 \\
\mathrm{am}\end{array}$ & $\begin{array}{c}12 / 12 / 01 \\
\mathrm{pm}\end{array}$ & $\begin{array}{c}13 / 12 / 01 \\
\mathrm{am}\end{array}$ & $\begin{array}{r}13 / 12 / 01 \\
\mathrm{pm}\end{array}$ & $\begin{array}{r}14 / 12 / 01 \\
\mathrm{am}\end{array}$ & $\begin{array}{c}14 / 12 / 01 \\
\mathrm{pm}\end{array}$ & $\begin{array}{c}15 / 12 / 01 \\
\mathrm{am}\end{array}$ & $\begin{array}{c}15 / 12 / 01 \\
\mathrm{pm}\end{array}$ & $\begin{array}{c}16 / 12 / 01 \\
\mathrm{am}\end{array}$ \\
\hline 1 & $\mathbf{6}$ & Rien & $\mathbf{5}$ & $\mathbf{5}$ & $\mathbf{4}$ & Rien & $\mathbf{5}$ & Rien & $\mathbf{6}$ \\
\hline 2 & Rien & Rien & Rien & Rien & Rien & Rien & Rien & $\mathbf{5}$ & 4 \\
\hline 3 & $\mathbf{5}$ & Rien & $\mathbf{5}$ & Rien & $\mathbf{5}$ & 7 & Rien & $\mathbf{5}$ & Rien \\
\hline 4 & Rien & Rien & Rien & $\mathbf{9}$ & 8 & $10^{* *}$ & Rien & $\mathbf{1 1}$ & Rien \\
\hline 5 & $4^{* * * *}$ & 3 & 2 & Rien & $4 * * * *$ & 5 & 7 & & \\
\hline
\end{tabular}

$\mathrm{X}$ : pas de mesure effectuée. Propriétaire 1:1 cochon Propriétaire $2: 2$ cochons Propriétaire $3: 2$ cochons Propriétaire $4: 5$ cochons Propriétaire $5: 3$ cochons

\author{
* plus $0.5 \mathrm{kgs}$ d'ordures. \\ ** plus $2 \mathrm{kgs}$ d'ordures. \\ *** plus $1.5 \mathrm{kgs}$ de patates douces cuites. \\ **** plus $2 \mathrm{kgs}$ d'ordures. \\ ***** plus $1 \mathrm{~kg}$ d'ordures. \\ $* * * * * * 2 \mathrm{kgs}$ d'ordures.
}

17. Traduction des auteurs. 
Quel est le lien entre le nombre de jardins ouverts et la quantité de cochons élevés? Nous ne croyons pas qu'il y ait une relation directe entre ces deux pratiques, même si une part des produits des jardins revient aux cochons. Si chez les populations Yali d'Irian Jaya (Boissière, 1999), les cochons vivent souvent en semi-liberté et reçoivent les patates douces des jardins récemment laissés en jachère et impropres à la consommation humaine, à Oksapmin, un jardin de billon succède à un autre, les villageois nourrissent les cochons avec une part des récoltes des jardins en activité et avec ce qui reste lorsqu'on détruit les anciens billons pour en faire de nouveaux. Concernant les jardins de pente, en revanche, on observe les mêmes pratiques que pour les Yali. Dans un cas comme dans l'autre, les tubercules donnés sont de petite taille (toan bap " petite patate douce »).

La nourriture donnée aux cochons fait l'objet de pratiques liées à leur élevage. En effet, on apprend aux cochons à ne pas manger de tubercules de taros, par exemple. Des boutures de taros avaient été plantées à l'intérieur de l'enclos à cochons et aucun cochon n'est venu les détruire, car ils n'ont pas été habitués à les consommer. Ce n'est pas le cas des cochons sauvages pour lesquels les taros sont une nourriture appréciée et la destruction des jardins de taros est une pratique courante.

Nourrir un cochon, c'est également chercher à le faire grossir. Il arrive que certaines pratiques de magie soient utilisées, en particulier par les membres du clan spécialisé dans les cochons. Autrefois, lorsqu'un cochon avait une taille particulièrement imposante, quand on venait de le tuer, un membre du clan du cochon allait chercher dans le corps du cochon une pierre de couleur sombre, qui serait située au-dessus du foie. Cette pierre, placée au-dessus d'un cochon lorsqu'il dormait dans sa porcherie, avait la propriété de faire grossir le cochon plus vite. La dernière pierre trouvée l'a été au début des années 1970, elle est encore utilisée de nos jours.

\section{Le don et le retour de don}

Trois personnages forment le noyau des relations qui s'établissent autour de l'élevage du cochon. Il est important de décrire ces trois personnages dont les rapports déterminent tout un réseau de relations entre les différents membres de la société et tout un jeu de pratiques liées à l'agriculture.

\section{Anmatin : le donneur}

Il s'agit d'un homme, d'une femme ou d'un enfant qui, possesseur de plusieurs cochons, souhaite en laisser un en dépôt chez un parent ou un affin pour que ce dernier l'élève. Si officiellement, l'anmatin offre son cochon parce qu'il n'a pas le temps de s'en occuper, la véritable raison est que ce geste lui permet de tisser des liens et des relations d'échange avec d'autres villageois n'appartenant souvent ni à son lignage ni à son clan. Le retour de ce don est le partage de la viande quand le cochon est tué, des porcelets s'il s'agit d'une truie ou le partage du prix de sa vente. Toute décision concernant le cochon offert (vente, consommation, don en compensation) doit être prise après concertation du receveur avec le donneur.

\section{Sahapat : le receveur}

C'est le gardien, celui qui s'occupera du cochon reçu de l'anmatin. Il le nourrit, le soigne, construit une porcherie pour lui. Il arrive que le sahapat soit choisi par l'anmatin. Parfois, le prétendant sahapat demande un cochon au futur anmatin. Lorsqu'un sahapat reçoit un cochon, il va passer beaucoup de temps avec son nouveau cochon, pendant une semaine environ, pour que ce dernier s'habitue à lui. Une relation est ainsi tissée avec l'objet de l'échange.

\section{Andah nita : la « sage femme »}

La personne qui a aidé à la naissance du sahapat est récompensée de son acte salissant, en recevant une part des cochons élevés par le sahapat. Cette personne est en général une femme, mais il arrive qu'un homme qui voulait être l'andah nita d'un nouveau-né participe à sa naissance, en construisant par exemple un abri pour que la mère puisse accoucher. Le statut d'andah nita par rapport à un sahapat est transmissible, généralement par les femmes. Cette fonction est donc plus liée à la nécessité de donner une part de porc qu'à une personne spécifique. L'andah nita ne reçoit un don de la part du sahapat que lorsque le cochon est tué.

Il faut toujours qu'il y ait une raison assez importante pour tuer un cochon : le paiement d'une compensation, la visite d'un parent éloigné ou alors la punition pour un acte que le cochon lui-même aurait commis, comme les dégâts que l'animal pourrait avoir fait dans un jardin et qui mérite le châtiment suprême. La participation au festin est une affaire de famille. On n'attache pas le cochon avant de l'exécuter. On va le chercher, s'il n'est pas dans l'enclos, et 


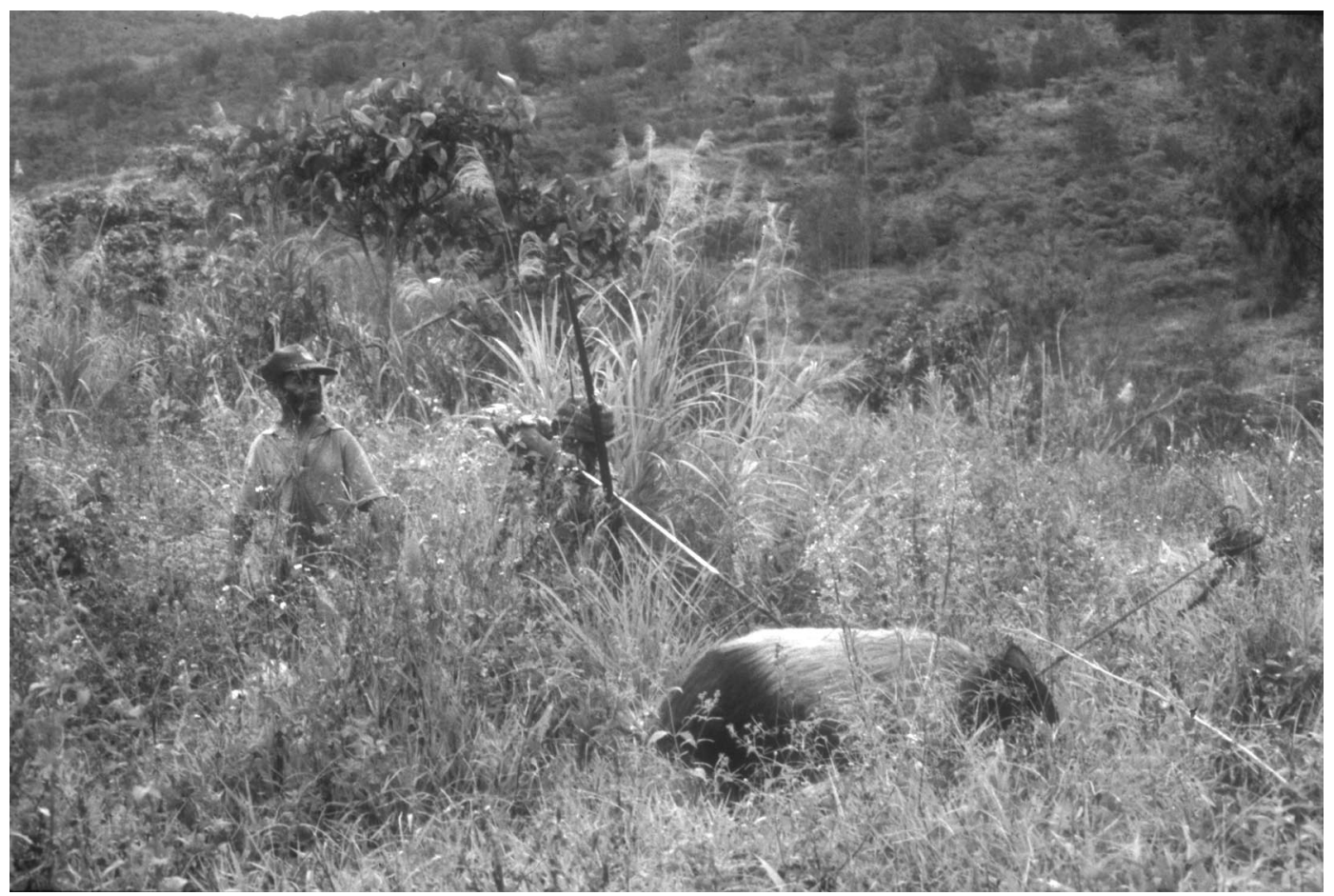

Рното 8. - Cochon abattu pour un mumu. Trangap-Oksapmin, 2001. (Manuel Boissière).

on l'amène devant son exécuteur qui le tue à l'arc en visant le cœur à courte distance (photo 8 ). $\mathrm{Si}$ l'archer est bon, il ne fera pas souffrir l'animal et une seule flèche suffira pour que l'animal meurt en quelques secondes. Ensuite, on passe l'animal entier sur le feu pour en brûler le poil et, une fois l'opération terminée, la phase la plus délicate commence, le découpage. Seuls des hommes expérimentés peuvent se livrer à cette chirurgie. Jadis débité à l'aide de tranchants en os (des vertèbres aiguisées de cochon) ou en bambou, depuis une trentaine d'année le découpage est rendu plus simple grâce à l'utilisation des tranchants en acier. Le dépeçage reste néanmoins exécuté selon le schéma traditionnel. L'animal est saigné et son sang est recueilli dans de larges feuilles et cuit pour en faire un boudin. Le dépeçage est réalisé de l'anus de l'animal vers sa gorge en parcourant la partie inférieure de son corps en direction verticale (photo 9). La carcasse est divisée en deux parties symétriques dans le sens de la longueur, la tête et la queue sont coupées à hauteur de la moitié de la colonne vertébrale. Les intestins et les organes internes sont extraits en faisant attention à ne pas faire éclater la rate ou répandre la bile sur la chair de l'animal. Les intestins sont donnés aux femmes pour qu'elles aillent les laver au ruisseau. Ensuite elles les cuisineront et les mangeront.
Le découpage de la carcasse du cochon ainsi que son partage et la distribution aux convives (photo 10), sont l'apanage du propriétaire de l'animal tué. Le boudin fait avec le sang est distribué indifféremment à tous.

Concernant l'échange qui se met en place entre les trois personnages, anmatin, sahapat et andah nita, il existe trois cas de figures selon que le cochon est tué ou vendu :

- Le cochon est tué et consommé : dans ce cas, il est partagé entre les trois personnages, selon des règles particulières. La tête, la moitié du corps, la moitié supérieure de la colonne vertébrale, une partie de l'intestin grêle et une partie du gros intestin reviennent à l'anmatin. L'autre moitié du corps, l'estomac, une partie du gros intestin, une partie de l'intestin grêle et la partie inférieure de la colonne vertébrale reviennent au sahapat. Enfin la cage thoracique (andah nita signifie " possesseur de la cage thoracique »), le cœur, les poumons et le foie reviennent à l'andah nita. Le partage est obligatoire entre l'anmatin et le sahapat. Toute tromperie serait grave et entraînerait le versement de compensations. L'oubli de la part revenant à l'andah nita semble être moins grave, ce dernier vivant parfois à plusieurs jours de marche du sahapat. 


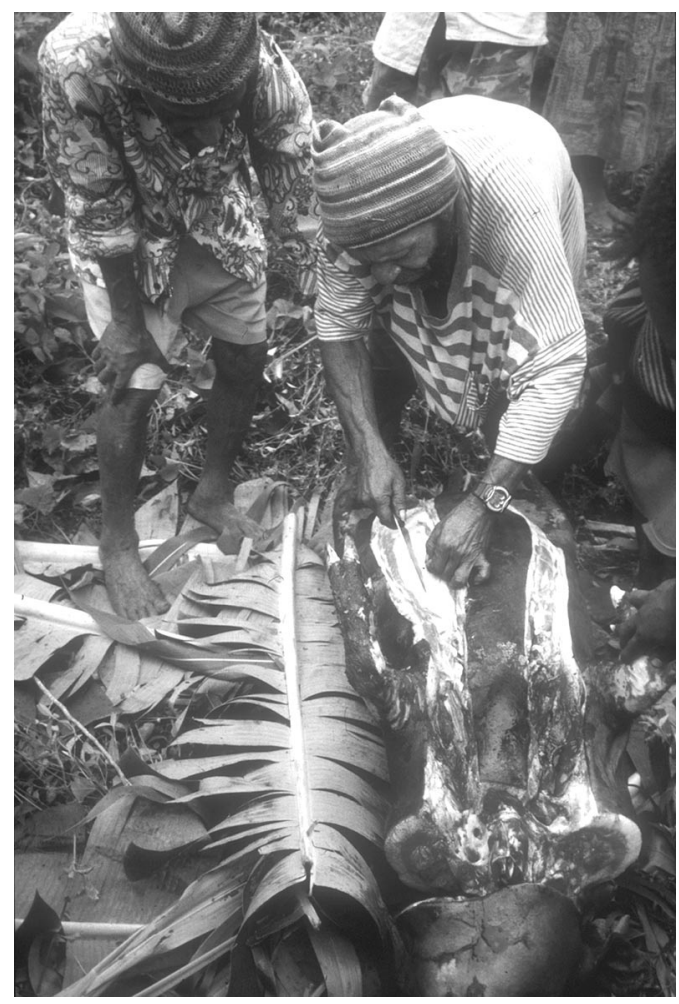

Рното 9. - Découpage du porc. Trangap-Oksapmin, 1998. (Lorenzo Brutti).

- Le cochon est vendu (vivant ou en morceaux) : le prix de la vente est partagé entre le sahapat et l'anmatin, l'andah nita ne recevant pas d'argent, mais éventuellement la part qui lui revient. Il arrive que le receveur soit négligeant et ne s'occupe pas du cochon donné par l'anmatin. Si l'anmatin continue de nourrir le cochon en principe donné, il sera le seul bénéficaire de sa vente et le sahapat perd alors tous ses droits.

- Le cochon est gardé en vie et sert à la reproduction : les porcelets qui naîtront seront partagés entre le donneur et le receveur. L'andah nita ne recevra de part que si un cochon est tué. Si par exemple la truie a trois porcelets, le sahapat en garde deux et en donne un à l'anmatin. La fois suivante, si la truie met à bas trois autres porcelets, c'est l'anmatin qui en recevra deux pour équilibrer. Les cochons qui reviennent au sahapat lui appartiennent de plein droit et ne sont plus sujet à partage. Libre à lui de les garder ou de devenir l'anmatin d'une nouvelle personne. Le propriétaire d'un cochon qui n'est soumis à aucune contrainte liée à des échanges est appelé musum, expression qui signifie une action faite sans rétribution.

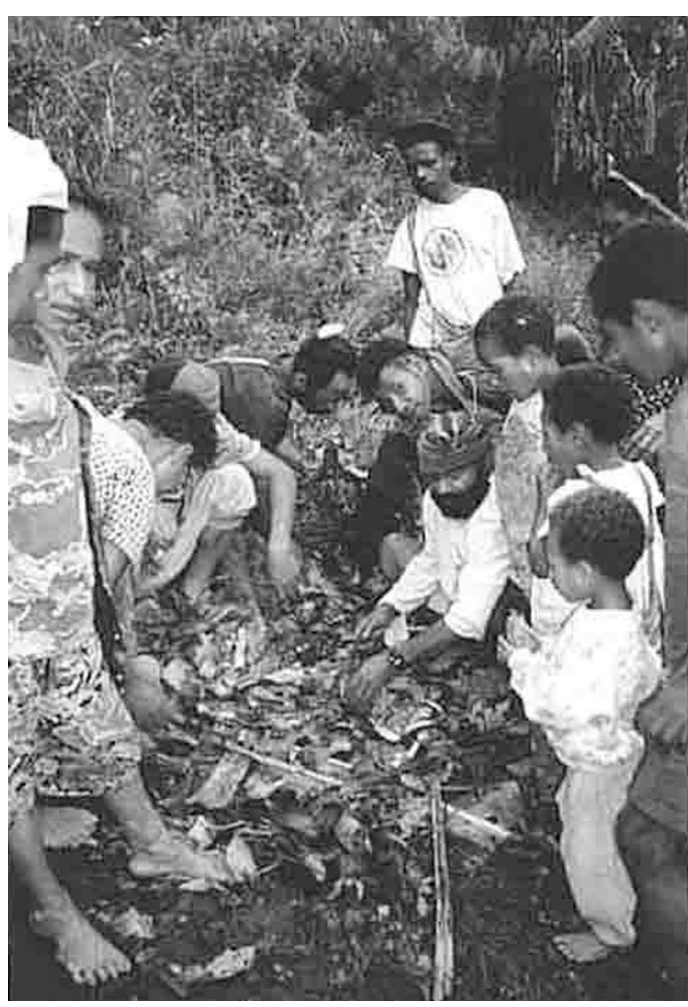

Рното 10. - Festin au hameau de Tambanaha. Trangap-Oksapmin, 1998. (Lorenzo Brutti).

Les seuls interdits à la consommation du cochon domestique concernent certains organes internes qui ne peuvent pas être mangés par les femmes et les enfants (photo 11). L'estomac, le foie et les reins du cochon leurs sont interdits alors qu'en tant qu'individus non initiés ils peuvent manger le cœur et les intestins. En ce qui concerne le cochon sauvage tué lors d'une sortie de chasse, après avoir consommé de sa chair, il faut s'abstenir d'aller dans les jardins pendant plusieurs jours. Cela sert à empêcher que la force vitale (äm) du sanglier, qui est liée au monde sylvestre et non domestiqué puisse causer des dommages dans un espace préalablement sauvage mais qui a désormais été aménagé par l'homme (ap).

\section{Conclusion}

Le cochon est encore aujourd'hui, malgré la monétarisation, un indice de prestige, sinon de richesse pour un homme. Cet animal domestique, sous sa double forme de porc et de cochon, mort ou vivant, constitue encore une monnaie d'échange complémentaire à la devise nationale officielle. Une partie des compensations doit être 


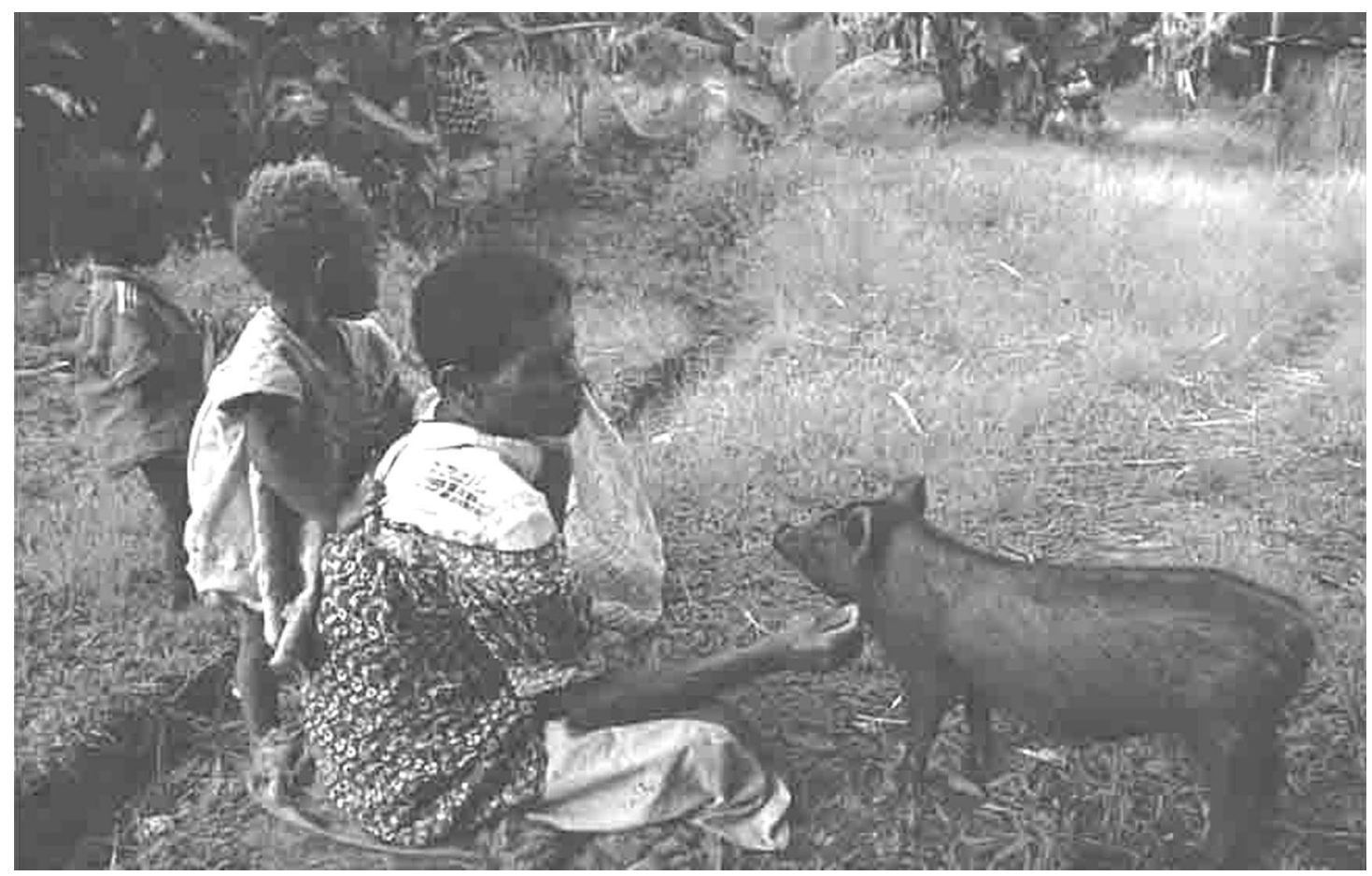

Рното 11. - Krimsau (décédée en 2000), la première femme de Disit Hilin, en compagnie de sa petite fille Valentine et de son cochon. Trangap-Oksapmin, 1997. (Lorenzo Brutti).

encore parfois payée en cochons. Cela montre l'importance de la transaction. Le cheptel de cochons est une des préoccupations principales pour un homme. D'autre part, une des formules de politesse des hommes qui se rencontrent le long des chemins est «comment vont tes cochons? », question qui permet à l'interlocuteur d'enchaîner sur d'autres sujets et de porter la discussion sur une série de thèmes liés à l'élevage, l'agriculture, la chasse, la vie quotidienne, le mariage, etc.

Une dernière remarque doit être faite concernant l'éloignement par rapport au monde de la nature, la distance instaurée entre les animaux, notamment des animaux de basse-cour, et l'espace domestique. Dans la maison traditionnelle oksapmin, l'accès des deux principaux animaux domestiques captifs, le cochon et le chien, est sinon permis indéfiniment, du moins admis et réglementé. Les chiens entrent à leur guise dans les maisons traditionnelles et en sont chassés seulement s'ils s'attaquent à la nourriture des hommes. La truie et ses petits, du moins pendant leurs premières semaines de vie, dorment souvent dans la maison à côté des hommes ou des femmes, pour les habituer à ne pas abandonner l'espace domestique du hameau et pour protéger leur santé fragile. Comme nous l'avons déjà souligné, il n'est pas rare de voir une femme donner le sein aux porcelets. Les cochons adultes malades ou qui se sauvent souvent en forêt sont aussi admis dans les maisons pour éviter qu'ils disparaissent dans la nature.

Cette cohabitation, ce partage homme/animal de l'espace domestique n'existe plus dans le contexte moderne des haus kapa ${ }^{18}$ où les chiens sont gardés à l'extérieur et les cochons confinés dans leurs enclos, mesure récemment imposé par les fonctionnaires du gouvernement pour limiter les dégâts commis dans les jardins et dans un souci d'hygiène. Les jeunes oksapmin considèrent aujourd'hui souvent avec dégoût la promiscuité d'antan entre les humains et les animaux et comme on le sait, les Adventistes vont jusqu'à s'interdire d'élever des cochons et d'en consommer la viande en s'installant eux-mêmes dans des enclos pour humains : les SDA kemp. Les camps des Adventistes du Septième Jour, se caractérisent en effet par une hygiène poussée à l'extrême où toute activité coutumière est interdite.

On assiste alors à une intéressante inversion de rôles en ce qui concerne la séparation entre 
nature et culture, représentées par la séparation de l'espace forestier et de l'espace domestiqué, démarquée par les enclos et les barrières de jardins. En fait, dans le système agricole oksapmin pré-européen, les jardins étaient entourés de barrières pour empêcher les incursions dévastatrices des cochons domestiques et sauvages. Récemment, la politique agricole nationale a poussé les paysans à enfermer leurs cochons dans des enclos assez larges pour que les betes puissent se promener à leur aise. Par contre les jardins, au moins ceux situés en basse et moyenne montagne, où l'on ne risque pas les incursions de cochons sauvages venant de la forêt, sont de plus en plus dépourvus de barrières. Cela pour plusieurs raisons : d'un côté les barrières sont moins indispensables car la présence de cochons errants en basses et moyennes vallées est de moins en moins probable; de l'autre côté, la diminution des périodes de jachère et l'exigence de créer des jardins de plus en plus nombreux en raison de la croissance démographique et de la demande du marché de Tabubil rendent moins nécessaire l'érection de barrières autour d'un nouveau jardin. De surcroît, la diminution des portions de forêts peuplées de grands troncs de vieux arbres qui constituaient la matière première pour ériger les barrières de jardins rend plus compliqué ce travail dans les jardins ouverts sur d'anciens recrus. La forêt - qui jadis constituait l'immense partie du territoire - ainsi que ses habitants étaient tenus à l'écart de l'espace domestiqué. De nos jours, les rôles se sont inversés : le monde animal représenté par les incursions des cochons qui, jadis, étaient écartés des espaces domestiqués grâce aux efforts humains, se trouve aujourd'hui clôturé et exclu de la domestication du territoire qui s'est étendue à une grande portion du paysage. On pourrait donc schématiser cette inversion des espaces de la façon suivante :

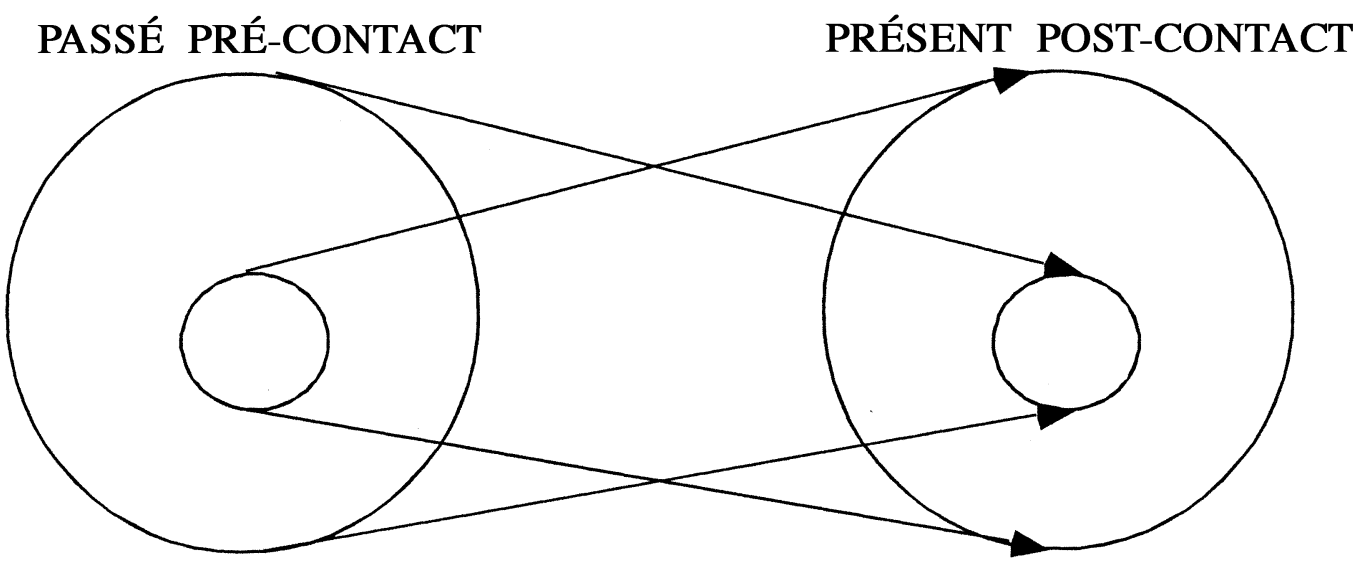

FIG. 1. - Transformations des oppositions spatiales entre territoire domestiqué et territoire non domestiqué (Source : L. Brutti)

L'espace domestiqué des jardins qui était clôturé et protégé de l'espace sauvage et des animaux se trouve aujourd'hui progressivement remplacé par des espaces dévolus à la vie animale semi-domestiquée qui sont maîtrisés, englobés et séparés. Les frontières de cet espace semidomestiqué élargi gagnent de plus en plus de terrain sur le territoire forestier.

Une dernière remarque en guise de métaphore. Les champs aseptiques et clos des Adventistes du Septième Jour qui se veulent affranchis $\mathrm{du}$ territoire forestier, et donc de la nature non maîtrisée, sont eux aussi entourés de barrières pour empêcher le passage des animaux tels que les cochons mais aussi, symboliquement, des êtres humains que sont les hommes et les femmes non convertis. Cette séparation spatiale propose alors une distinction symbolique entre sauvage et domestiqué, entre sylve et jardin, entre païen et converti, entre le milieu dominé par les forces de la nature et l'espace maîtrisé par les nouvelles lois de l'homme. Mais aussi, exactement comme les cochons ont été enfermés dans des enclos à cochons par la nouvelle politique écologique gouvernementale, les chrétiens Adventistes s'enferment dans des enclos pour humains du fait de la nouvelle confession religieuse à laquelle ils sont convertis. Ce parallélisme inconscient dans l'inscription spatiale des cochons domestiqués et des humains relève donc symbolique- 
ment d'une transformation mais aussi d'une survivance de l'ancienne homologie entre cochon et personne humaine.

\section{RÉFÉRENCES}

BArrau, J., 1990. Les hommes dans la nature. Esquisse d'une histoire naturelle des sociétés et des mœurs humaines, in Histoire des Mours 1. Les coordonnées de l'homme et la culture matérielle. Paris, Encyclopédie de la Pléiade, Gallimard, pp. 9-58.

_, 1975. Écologie, in R. Cresswell, H. Balfet, J. Barrau, Ch. Bromberger, M. Godelier, G. RavisGiordani (eds.), Éléments d'ethnologie 2. Six approches. Paris, Armand Colin.

—,1973. Plantes et comportements des hommes qui les cultivent. L'œuvre ethnobiologique d'André Haudricourt, La Pensée 171, pp. 37-46

BoIssiÈRE, M., 1999. Ethnobiologie et rapports à l'environnement des Yali d'Irian Jaya (Indonésie), thèse université de sciences, Montpellier, $456 \mathrm{p}$.

BRUTTI, L., 1997. Waiting for God. Ecocosmological Transformations among the Oksapmin, in Andrew Strathern and Pamela Stewart (eds.), Millennial Markers in the Pacific. Townsville : Center for Pacific Studies, James Cook University of North Queensland.
—, 1999. 'Après nous le déluge'. Les effets de la présence d'une compagnie minière sur le système agricole et le commerce des produits cultivés dans la région de Oksapmin (Papouasie Nouvelle-Guinée), $J A T B A$, Revue d'ethnobiologie, 41(1), pp. 183200.

DiAmond, J., 1997. Guns, germs, and steel, W.W. Norton \& Compagny, New York, 480 p.

DWYER P.D., 1990. The pigs that ate the garden, The University of Michigan Press, Ann Arbor, 241 p.

—, 1996. Boars, barrows, and breeders : the reproductive status of domestic pig populations in mainland New Guinea, Journal of Anthropological Research, 52, pp. 481-500.

Haudricourt, A., 1972. Recherches d'ethnohistoire dans les archipels de l'Océanie Pacifique. Courrier du CNRS, 5, p. 38.

LEMONNIER, P., 1993. Le porc comme substitut de vie : formes de compensation et échanges en NouvelleGuinée, Social Anthropology, 1, 1A, pp. 33-55.

RAPPAPORT, R.A., 1970 (1968). Pigs for the ancestors. Ritual in the Ecology of a New Guinea People, Yale University Press, New Haven and London, $311 \mathrm{p}$.

SteEnsBerg, A., 1980. New Guinea gardens. A study of husbandry with parallels in prehistoric Europe, Academic Press, London, 222 p.

Strathern A., 1971. The Rope of Moka, Canberra, Australian National University. 